\title{
Manual-Based Group Intervention for Siblings and Parents of Children with Neurodevelopmental Disorders in Cambodia
}

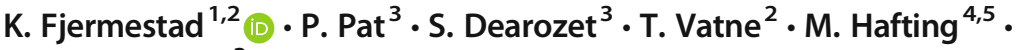 \\ B. Jegannathan ${ }^{3}$
}

Accepted: 20 October 2020/ Published online: 7 November 2020

(C) The Author(s) 2020

\begin{abstract}
Siblings of children with neurodevelopmental disorders (ND) are at increased risk of mental health problems. The burden on families of children with ND is exacerbated in low-income countries with limited health services and dependency on informal care systems. Yet, there is little research on family impacts of ND in non-Western settings, and no evidence-based interventions for siblings. We examined initial outcomes and feasibility of a manual-based intervention for siblings and parents of children with ND, called SIBS, delivered in Cambodia. SIBS has promising evidence from an open trial in Norway. We delivered eight groups for 52 siblings $(M$ age $=12.7$ years, $S D=2.7$; $44.0 \%$ female) and 56 caregivers ( $M$ age $=43.5$ years, $S D=8.5 ; 61.1 \%$ mothers $)$ of 54 children with ND at the only public child mental health clinic in Cambodia. We aimed to improve sibling and parent mental health and family communication. The SIBS intervention comprises three separate sibling/parent group sessions and two joint sibling-parent dialogue sessions. Parent-reported mental health scores for siblings were higher than sibling self-report. Parent mental health problems at baseline were high, with no difference between mothers and fathers. There was significant improvement in parent mental health and parent-rated mental health for siblings from baseline to 4month post-intervention (effect sizes $d=0.44$ to 0.52 ). There was no change in siblingreported mental health or family communication. Sibling- and parent-rated user satisfaction was high. We conclude that the SIBS intervention showed promise in Cambodia. However, revision of the communication component is needed.
\end{abstract}

Keywords Developmental disorders · Siblings · Intervention · Low and middle income countries

\section{K. Fjermestad}

kristefj@uio.no

Extended author information available on the last page of the article 
Neurodevelopmental disorders (ND) affect at least 15\% of children (Thapar et al. 2017). ND involve impaired development of the central nervous system and affect numerous areas of the child's functioning including language, behavior, sleep, physical abilities, and mental health. Examples include autism, intellectual disability, and cerebral palsy. ND in children require repeated and time-consuming follow-up from parents in the home, in the child's education setting, and in clinics. Families of children with ND experience complex burdens including time and financial restraints, social stigma and isolation, guilt and grief, uncertainty and unpredictability, loyalty conflicts, parental (dis) stress and depression, and potential trauma (Haukeland et al. 2015; Karst and Van Hecke 2012). A unique risk profile of multiple concurrent and potentially accumulating risk factors faces these families. Although childhood ND represent a global challenge, the risks associated with ND are exacerbated for families of children with ND in developing, low to middle income and/or emerging economy countries with limited or inaccessible health services for children with ND. Nevertheless, there is much less research on ND in low-income countries, and research on families of children with ND in such contexts is needed.

Childhood ND represent strain for parents, and also particular challenges for sibling relationships, life's longest lasting familial bonds. Siblings of children with ND (herein, siblings) experience multiple burdens. These include extra care responsibilities; worries and confusion; reduced coping, resilience, and social support; and impaired family communication (Murphy et al. 2017; Tudor and Lerner 2015; Vermaes et al. 2012). As a consequence, siblings are at increased risk of depression, anxiety, behavior problems, and impaired quality of life, self-concept, social support, resilience, and coping (Haukeland et al. 2015; Tudor and Lerner 2015; Vermaes et al. 2012). Longitudinal studies of siblings further show the increased risk is persistent across childhood and into adulthood (Fisman et al. 2000; Giallo et al. 2014). In low to middle income and emerging economy countries, the strain on siblings may be even greater due to increased informal care responsibilities. The mental health risks identified among siblings hold huge financial burdens on society, including school/work absence and health service needs (Knudsen et al., 2010). Secondary prevention of mental health disorders in this atrisk group can promote health and resilience in siblings - and save public health spending via the substantial care tasks siblings provide in their families. This also promotes health and wellbeing among persons with ND (Rossiter and Sharpe 2001).

Despite the documented risks for siblings, evidence-based interventions for this population are lacking. Existing interventions typically involve sibling groups, and some studies have documented positive outcomes of sibling interventions on mental health symptoms, stress, self-esteem, social support, and sibling relations (Smith and Perry 2005; Phillips 1999; Williams et al. 2003). Although these findings are important, existing studies have limitations, and none have sufficient methodological quality to qualify as "well-established" or "probably efficacious" (McKenzie Smith et al. 2018; Tudor and Lerner 2015). Limitations include lack of control conditions; sufficient power (i.e., small sample sizes); inclusion criteria beyond being siblings of children with disorders; use of standardized measures; manuals or sufficient session content description; sufficient description of parent involvement when featured; empirically and conceptually based predictors of outcome; and follow-up data (Tudor and Lerner 2015). Furthermore, existing interventions are time-consuming for families and health providers, typically requiring 10-12 session hours over several weeks. Thus, 
participation may represent additional strain for already challenged families. Outside research settings, support groups for siblings may be offered as part of activity-based events such as camps, often by non-professional volunteers with limited training. Although potentially useful and enjoyable for siblings, there is no documentation of the effects of such groups. There is also limited consideration of the potential negative effects talking about difficulties in support groups may have on siblings, which trained health providers may be more equipped to handle.

Existing interventions are primarily developed for and delivered in high-income Western countries, based on Western cultural norms and health services. The latest review on intervention for siblings included 17 studies, of which none were conducted outside Australia, Europe, or USA (McKenzie Smith et al. 2018). The extent to which programs tailored for siblings exist in emerging economies is unknown. In the current study we address this gap, by evaluating initial outcomes and feasibility of a brief group intervention for siblings and parents of children with ND developed in Norway, when delivered in Cambodia. The intervention is called SIBS; and has initial evidence from an open trial with 99 families in Norway (Haukeland et al. 2020). SIBS has been developed in close collaboration with several user associations and is based on several years of sibling work by a Norwegian consortium (Vatne et al. 2019). SIBS is manualbased and comprises five sessions, usually delivered over 2-3 days in the course of a week. Three of the sessions are parallel (but separate) groups for parents and siblings. The remaining two sessions are joint sessions in which each sibling talk to their parent alone. The main topics are siblings' understanding of the ND, and their perception of family challenges. Parents are taught communication skills and encouraged to listen, explore, and validate in response to siblings' questions about the ND and their ideas about family challenges. The intervention is based on a theoretical framework for resilience development in children, focused on the importance of relations (i.e., the dyadic dialogue component) and sense of competence (i.e., understanding of the ND and related challenges; Sommerschild 1998).

The project presented in the current paper is the result of a longstanding collaboration between Norwegian and Cambodian professionals (Hafting et al., 2020). Cambodia is a South-East Asian country which shares borders with Thailand to the (north) west, Vietnam to the east, and Laos to the northeast. According to the latest numbers from the World Health Organization (WHO), the gross national income per capita is 2890 USD, while the total expenditure on health per capita is 183 USD (WHO 2020a). In comparison, the same numbers for Norway, where the SIBS intervention was developed, is a gross national income per capita of 66,520 USD, while the total expenditure on health per capita is 6347 USD (WHO 2020b). As such, the health contexts are extremely different in the two settings.

Cambodia has a very disruptive recent history, with the genocide committed by the Pol Pot regime in the 1970s followed by war with Vietnam until the early 1990s. Thus, Cambodia is a post-conflict and low-income country, recovering from three decades of war and civil unrest. Theravada Buddhism is the official religion of Cambodia, and there is a strong influence of animism and Brahmanism which may influence the people's beliefs and attitudes towards disabilities. The Paris Peace Agreement in 1991 brought stability and democratic governance as well as international influence through nongovernmental and other agencies who try to revive the health care system, which was totally destroyed during the Khmer Rouge era (Grundy et al. 2009). The effort to rebuild 
the medical services based on a Western perspective may have undermined the traditional belief system and promoted biomedical views on health and disability. In a qualitative study, Morgan and Tan (2011) reported that an equal numbers of Cambodian caregivers attributed the cause of their child's disability to biomedical issues as to traditional views relating to Buddhistic and animistic views. While the parental understanding and attitudes towards disabilities might be influenced both by religion and current prevailing Western notions of biomedical causality, siblings may in turn be influenced by the members of the multigenerational family as grandparents share the same house or live quite close-by. A study which addressed intergenerational transmission of trauma in Cambodia reported how parents and grandparents may use their experiences, beliefs, and attitudes to discipline their children in the process shaping their belief system on health, trauma, and disabilities (Melander et al. 2016).

At the same time, the younger generation in Cambodia has improved English skills and vast access to online information (Clayton 2017; Telecommunication Regulator of Cambodia 2020), which may expose them to different information and attitudes to disabilities compared to the parental generations above them. During the last decades, Cambodia's economy has undergone an average $8 \%$ growth rate, which makes the country one of the world's fastest-growing economies (World Bank 2020). There is an increase in romantically based marriages, divorce rates, and job-related family separations, while birth rates are decreasing and young people leave the parental home earlier (Heuveline and Hong 2017). As such, Cambodian society is in an exciting and fastmoving transition, and maneuvering a Western intervention to fit the Cambodian family and generation culture is a complex venture.

The Cambodian setting in which the SIBS intervention was tried in the current study is the Caritas Chey Chumneas Child and Adolescent Mental Health Clinic (CaritasCCAMH), the only child and adolescent mental health clinic in Cambodia. CaritasCCAMH is a public-private partnership between Ministry of Health Royal Government of Cambodia and Caritas Cambodia which started in 1995. This multi-disciplinary clinic provides clinical services for children and adolescents with mental health and neurodevelopmental disorders, including group interventions (Jegannathan et al. 2014).

In the current study, we have three main aims. First, we describe the level of sibling and parent mental health and family communication among our sample of Cambodian siblings and parents. This is to provide the field with initial knowledge about potential service needs for this population. Second, we examine change in mental health symptoms for siblings and parents, as well as perceived family communication from baseline to 4-month follow-up. This is to evaluate initial outcomes of SIBS when delivered in Cambodia. Third, we examine sibling and parent perceived usefulness and satisfaction with the SIBS intervention, with the aim to evaluate initial feasibility of SIBS in this cultural context.

\section{Methods}

\section{Sample}

The sample comprised 108 participants, i.e., 56 caregivers and 52 siblings from 54 families. The siblings were aged 8 to 21 years $(M=12.7, S D=2.7 ; 44.0 \%$ female). 
Each sibling was accompanied by at least one caregiver (61.1\% mothers, $20.4 \%$ fathers, $9.3 \%$ grandmothers, $7.4 \%$ both parents, and one older sister $1.8 \%$ ). The caregivers were aged 21 to 75 years $(M=43.5, S D=8.5)$. In terms of caregiver education, $22.5 \%$ of fathers and $13.6 \%$ of mothers had college or University education. Some parents had no schooling at all, i.e., $7.5 \%$ of fathers and $2.3 \%$ of mothers. Caregivers rated their family economy as good $(11.9 \%)$, medium $(54.8 \%)$, or poor $(33.3 \%)$. The mean number of children per family was $3.3(S D=2.5)$. All families had at least one child with a diagnosis of learning problems $(31.2 \%)$, intellectual disability $(18.8 \%)$, autism spectrum disorder (16.7\%), attention deficit hyperactivity disorder $(12.5 \%)$, developmental delay $(10.4 \%)$, or Down syndrome $(10.4 \%)$. The mean age of the child with disorder was 9.6 years (range 3 to 16 years, $56.0 \%$ male).

\section{Procedures}

All families were enrolled at the Caritas-CCAMH, with the child with ND as the primary patient. Families were invited by clinic staff by phone to take part in the SIBS intervention, with all five sessions delivered in one day. Only three approached families declined. Two declined due to exam periods for the sibling, one family declined due to overseas travels. It was made clear that participation was voluntary and that lack of consent would not affect health services offered to the family. When families arrived in the morning, they signed consent forms and completed questionnaires, with reading/ writing assistance from clinic staff if needed. Siblings and parents completed questionnaires in separate rooms. Sessions 1-3 were delivered before lunch, and sessions 4-5 were delivered after lunch. If required, clinic assistants looked after the child with disorder during the intervention. Money for lunch and transportation was covered by the clinic. The CCAMH clinic is located in the town of Takhmao, Kandal Province, approximately $15 \mathrm{~km}$ south of Cambodia's capital city, Phnom Penh. The longest travel time for participants to the clinic was about $5 \mathrm{~h}$ (median $2.5 \mathrm{~h}$ ). Transportation was arranged for those families who lived furthest away. The project was approved by the National Committee for Research Ethics in Cambodia, and by the Regional Committee for Research Ethics in South-Eastern Norway. In accordance with the ethical approval, participants were informed that the research team could help with further intervention for the sibling or parent, if needed. No extraordinary measures were deemed necessary. However, all participating families continue to receive support from Caritas-CCAMH as required based on family needs.

\section{Intervention}

See Fig. 1 for overview of the intervention. The main aim of the group intervention is to train parents in communication skills (i.e., how to listen, explore, and validate when talking to their child) and to provide siblings with an opportunity to discuss the disorder of their brother/sister, and associated emotions and family challenges. In the current study, the five intervention sessions were delivered over one day. Sessions 1, 2, and 4 are parallel (separate) group sessions for siblings and parents. Sessions 3 and 5 are integrated sibling-parent dialogues in which each sibling and parent talk together in pairs. Session 1 is introduction. Sessions 2-3 focus on siblings' disorder knowledge based on a semi-structured group interview (Lobato and Kao 2002). Sessions 4-5 focus 


\begin{tabular}{|c|c|c|c|c|c|}
\hline & $\begin{array}{l}\text { Session } 1 \\
20 \mathrm{~min} .\end{array}$ & $\begin{array}{c}\text { Session } 2 \\
60 \mathrm{~min} .\end{array}$ & $\begin{array}{c}\text { Session } 3 \\
60 \mathrm{~min} .\end{array}$ & $\begin{array}{c}\text { Session } 4 \\
60 \mathrm{~min} .\end{array}$ & $\begin{array}{c}\text { Session } 5 \\
60 \mathrm{~min} .\end{array}$ \\
\hline Siblings & $\begin{array}{l}\text { Introduction } \\
\text { Aim: Getting to } \\
\text { know each other/ } \\
\text { the intervention. }\end{array}$ & $\begin{array}{l}\text { The disorder } \\
\text { Aim: Share } \\
\text { thoughts about } \\
\text { the disorder. } \\
\text { Write question(s) } \\
\text { to parents. }\end{array}$ & $\begin{array}{l}\text { JOINT } \\
\text { Siblings + Parents } \\
\text { Aim for siblings: Present } \\
\text { their questions to } \\
\text { parents. } \\
\text { Aim for parents: Practice }\end{array}$ & $\begin{array}{l}\text { Emotions } \\
\text { Aim: Talk about } \\
\text { emotions and } \\
\text { challenges. Write } \\
\text { challenge(s) to } \\
\text { discuss with }\end{array}$ & $\begin{array}{l}\text { JOINT } \\
\text { Siblings + Parents } \\
\text { Aim for siblings: Present } \\
\text { their challenges to } \\
\text { parents. } \\
\text { Aim for parents: Practice }\end{array}$ \\
\hline Parents & $\begin{array}{l}\text { Introduction } \\
\text { Aim: Provide } \\
\text { information about } \\
\text { the intervention. }\end{array}$ & $\begin{array}{l}\text { The disorder } \\
\text { Aim: Learn to } \\
\text { listen, explore, } \\
\text { and validate } \\
\text { siblings' thoughts } \\
\text { about the } \\
\text { disorder. }\end{array}$ & $\begin{array}{l}\text { taught in previous } \\
\text { sessions. } \\
\text { Joint goal: A positive } \\
\text { conversation experience. } \\
\text { Group leaders provide } \\
\text { brief supervision to each } \\
\text { dyad. }\end{array}$ & $\begin{array}{l}\text { Emotions } \\
\text { Aim: Learn how to } \\
\text { listen, explore, } \\
\text { and validate } \\
\text { siblings' } \\
\text { challenges. }\end{array}$ & $\begin{array}{l}\text { taught in previous } \\
\text { sessions. } \\
\text { Joint goal: A positive } \\
\text { conversation experience. } \\
\text { Group leaders provide } \\
\text { brief supervision to each } \\
\text { dyad. }\end{array}$ \\
\hline
\end{tabular}

Fig. 1 Overview of the SIBS intervention. All sessions were conducted in one day with a 2-h lunch break

on siblings' emotional experiences based on cognitive-behavioral principles about how thoughts and behavior influence emotions. In Sessions 2 and 4, siblings prepare lists of questions about the disorder and experienced family challenges, respectively, which a group leader brings to the parent group for discussion. In Sessions 3 and 5, each sibling discusses the questions and challenges with their parent. The main component of the parent sessions is communication training, using standardized video examples of real sibling-parent dialogues with various degrees of open-exploratory communication as basis for discussion. The parent sessions also comprise psychoeducation about sibling challenges, and introduction to cognitive-behavioral principles about emotional coping by regulating thoughts and behaviors. The intervention is outlined in a step-by-step manual for group leaders. The original SIBS manual is in Norwegian, and was translated to English by one of the authors. The English version was used in training and supervision, and an abbreviated Khmer version was developed by the CaritasCCAMH team. All versions (Norwegian, English, Khmer) are available upon request to the first author.

\section{Group Leaders}

Eight groups with 5 to 9 participating families each were delivered. See Table 1 for overview. Group leaders were regular clinical staff (i.e., psychologists, social workers, psychiatrists, and an art therapist) who volunteered to participate. The training of group leaders comprised four main steps. First, they attended a two-day introduction to the intervention comprising lectures and role plays. Second, they conducted five pilot groups at Caritas-CCAMH, with written and verbal feedback from participants. Third, experiences from the pilot was discussed between the Cambodian and the Norwegian teams in preparation for the study. Fourth, the two-day training in the intervention was repeated, and video examples and written materials for the participants were prepared in Khmer.

Throughout this training process, we held discussions concerning cultural adaptation, meaning of concepts, how to present intervention contents in ways that were meaningful in a Cambodian culture. These workshops were based on video clips from Norwegian sibling groups (translated), presentation of results from an open trial in Norway (Haukeland et al. 2020), followed by team discussions and role plays in groups 
Table 1 Overview of groups for siblings and parents of children with neurodevelopmental disorders

\begin{tabular}{llcc}
\hline Group & Disorder (n) & Sibling age range (years) & \% attending follow-up \\
\hline 1 & Down syndrome (5) & $11-14$ & $40.0 \%$ \\
2 & ADHD (6) & $9-16$ & $100.0 \%$ \\
3 & Autism (8) & $8-13$ & $62.5 \%$ \\
4 & Intellectual disability (9) & $11-17$ & $77.8 \%$ \\
5 & Developmental delay (5) & $10-21$ & $80.0 \%$ \\
6 & Developmental delay (6) & $8-16$ & $50.0 \%$ \\
7 & Learning problems (8) & $8-15$ & $75.0 \%$ \\
8 & Learning problems (7) & $8-18$ & $57.1 \%$ \\
Total & N $=54$ & $8-21$ & $68.5 \%$ \\
\hline
\end{tabular}

$\mathrm{ADHD}=$ attention deficit hyperactivity disorder

with Caritas-CCAMH staff. Further adaptations were made based on the five pilot groups (unpublished and not part of the data presented herein), for example in terms of practical delivery. During the pilot, sessions 1-3 were delivered in one day, with sessions 4-5 delivered the following week - which is the current SIBS procedure in Norway. However, we experienced that it was tricky for many families to attend both days, and had no-shows both on the first and the second day of the intervention during the pilot. Therefore, we decided to re-arrange the schedule and arrange all five sessions in one day, to prevent dropout and/or families turning up on the second day only. Furthermore, considerable time was set aside before group start for families to complete study questionnaires. Adaptations to written materials (wording, precisions) were also made to Khmer versions of the intervention material based on feedback from the unpublished pilot groups.

\section{Measures}

The Strengths and Difficulties Questionnaire (SDQ; Goodman 2001) was used to measure sibling mental health status. The 25 -item SDQ was rated by siblings (selfreport), and by the participating sibling's caregiver(s) as proxy-reports on sibling wellbeing. The SDQ covers five domains (emotional, conduct, inattention, and peer problems, prosocial behavior). The SDQ has demonstrated excellent psychometric properties and overlap with lengthier mental health measures (e.g., Vostanis 2006). In the current study, internal consistency (Cronbach's alpha) for the SDQ was acceptable (Siblings $\alpha=.64$; Mothers $\alpha=.63$; Fathers $\alpha=.75$ ).

The Depression Anxiety Stress Scale (DASS; Lovibond and Lovibond 1995) was used to assess parental mental health. The DASS was completed separately by mothers and fathers. The DASS comprises 21 items rated on a 4-point scale from 0 (hardly ever) to 3 (almost always). The items cover parents' self-rated anxiety, depression, and tension-stress symptoms. The DASS has demonstrated satisfactory reliability, and convergent and discriminant validity (Lovibond and Lovibond 1995). In the current study, internal consistency (Cronbach's alpha) for the DASS was good (Mothers $\alpha=.93$; Fathers $\alpha=.96$ ). 
The Parent Child Communication Scale - child and parent versions (PCCS-C/P: Loeber et al. 1998) was used to measure family communication. The 10-item PSSC-C was rated by siblings about both parents (i.e., twice). The 20 -item PSSC-P was rated by caregivers. The PSSC-C/P has demonstrated good psychometric properties with Chronbach's $\alpha$ from .75 to .82 for PCCS (Haukeland et al. 2020; Loeber et al. 1998). In the current study, internal consistency (Cronbach's alpha) was acceptable (Siblings about mothers $\alpha=67$; Siblings about fathers $\alpha=.71$; Mothers $\alpha=.69$; Mothers $\alpha=.70$ ).

We used the official Khmer version of the SDQ, which has been translated by the Caritas-CCAMH team and is available on the SDQ website (www.sdqinfo.org). The other questionnaires were translated to Khmer from English by a member of the CaritasCCAMH team who trained as a Psychologist in Australia, and thus is fluent in both Khmer and English. Adaptations were made to item wordings based on participant feedback from the pilot groups. Back-translations of the questionnaires were checked and approved by the first author, who is Norwegian but has several years of clinical and research experience from English-speaking countries (United Kingdom, Australia) and who practices oral and written English daily as part of his academic position.

\section{Data Analytic Plan}

Descriptive analyses (means, frequencies, correlations) and paired-sample t-tests were conducted with IBM Statistics SPSS 25. Effect size differences between groups and from baseline to post were calculated as Cohen's d using the formula $\left(\mathrm{M}_{\mathrm{Group} 1}-\mathrm{M}\right.$ Group2)/SD pooled (Cohen 1992).

\section{Results}

\section{Description of Baseline Data}

See Table 2 for sibling and parent mental health and family communication baseline levels. Mothers and fathers rated siblings' mental health similarly; both significantly higher than sibling self-report $(p<.001)$. There was no difference between mother- and father-reported mental health $(p=.905)$. Siblings rated their communication with mothers significantly higher than their communication with fathers $(p<.05)$. There was no difference in the quality of family communication rated by mothers and fathers $(p=.434)$.

See Table 3 for correlations between the baseline variables. There were very few significant correlations. Sibling-rated mental health was not associated with any of the other variables. From the perspective of sibling-ratings, the only significant correlation was a large association in perceived communication with mothers versus fathers. Mother-rated mental health was significantly associated with mother-rated mental health for siblings and mother-rated communication with siblings.

\section{Outcomes}

Post-intervention data (4 months) was available from 37 caregivers and 33 siblings from 40 families (i.e., for $74.1 \%$ of participating siblings). There were no baseline 
Table 2 Baseline symptoms among 56 caregivers and 52 siblings in a caregiver-sibling intervention

\begin{tabular}{|c|c|c|c|c|}
\hline \multirow[t]{2}{*}{ Variable } & \multicolumn{3}{|l|}{$\mathrm{M}(\mathrm{SD})$} & \multirow[t]{2}{*}{ Scale range } \\
\hline & Total & Male & Female & \\
\hline Sibling mental health & & & & $0.0-40.0$ \\
\hline Self-report & $12.3(4.7)$ & $11.9(4.1)$ & $12.6(5.7)$ & \\
\hline Mother-report & $18.2(4.2)$ & $18.1(4.0)$ & $17.8(4.6)$ & \\
\hline Father-report & $17.9(4.8)$ & $17.7(5.9)$ & $19.3(2.1)$ & \\
\hline \multicolumn{5}{|l|}{ Caregiver mental health } \\
\hline Total & $25.0(13.9)$ & $22.6(13.5)$ & $25.7(14.1)$ & $0.0-63.0$ \\
\hline Depression & $8.0(5.1)$ & $6.3(4.3)$ & $8.4(5.1)$ & $0.0-21.0$ \\
\hline Anxiety & $7.6(5.0)$ & $7.6(4.5)$ & $7.7(5.1)$ & $0.0-21.0$ \\
\hline Stress & $9.3(5.0)$ & $8.8(5.3)$ & $9.6(4.9)$ & $0.0-21.0$ \\
\hline Family communication & & & & $1.0-5.0$ \\
\hline Child about mother & $3.0(1.1)$ & $3.1(1.0)$ & $2.7(1.2)$ & \\
\hline Child about father & $2.6(1.0)$ & $2.6(1.1)$ & $2.6(1.0)$ & \\
\hline Mother about child & $3.2(0.5)$ & $3.0(0.4)$ & $3.3(0.5)^{*}$ & \\
\hline Father about child & $3.3(0.5)$ & $3.2(0.6)$ & $3.4(0.2)$ & \\
\hline
\end{tabular}

Gender difference is significant at the $p<.05$-level

differences between participants who completed follow-up measures versus those who did not complete follow-up assessment, with one exception. Mothers who completed follow-up had more mental health problems at pre-intervention than those who did not attend follow-up, with a large effect size difference (0.68-point difference on 0-3 scale; $p=.014 ; d=1.15$ ).

There was a significant reduction in parent-reported sibling mental health, as well as in parent self-reported mental health from baseline to 4-month follow-up. Parentreported SDQ had been reduced with 2.3 points $(\mathrm{SD}=4.7)$ on a $0-40$ scale, an effect size difference of $d=0.52$. Parent mental health symptoms had been reduced with 6.3 points $(\mathrm{SD}=14.7)$ on a $0-63$ scale, an effect size difference of $d=0.44$. There was no significant difference in sibling-reported mental health or family communication (data not shown).

\section{User Satisfaction}

Immediately post-intervention, siblings and caregivers were asked to rate their satisfaction with the intervention. The evaluations were overall positive. See Table 4 for details. The evaluation forms included open-ended questions about what the participants wanted to be different (i.e., contain more or less of) in the intervention.

From 55 open-ended responses from siblings about what should be done differently, $24(43.6 \%)$ concerned participants' behavior and involvement in the group (e.g., We shouldn't interrupt each other when someone is talking), six (10.9\%) concerned group contents (e.g., We should discuss more about daily problems), five (9.1\%) concerned wishing there was more time for the groups (e.g., We should have this program more 


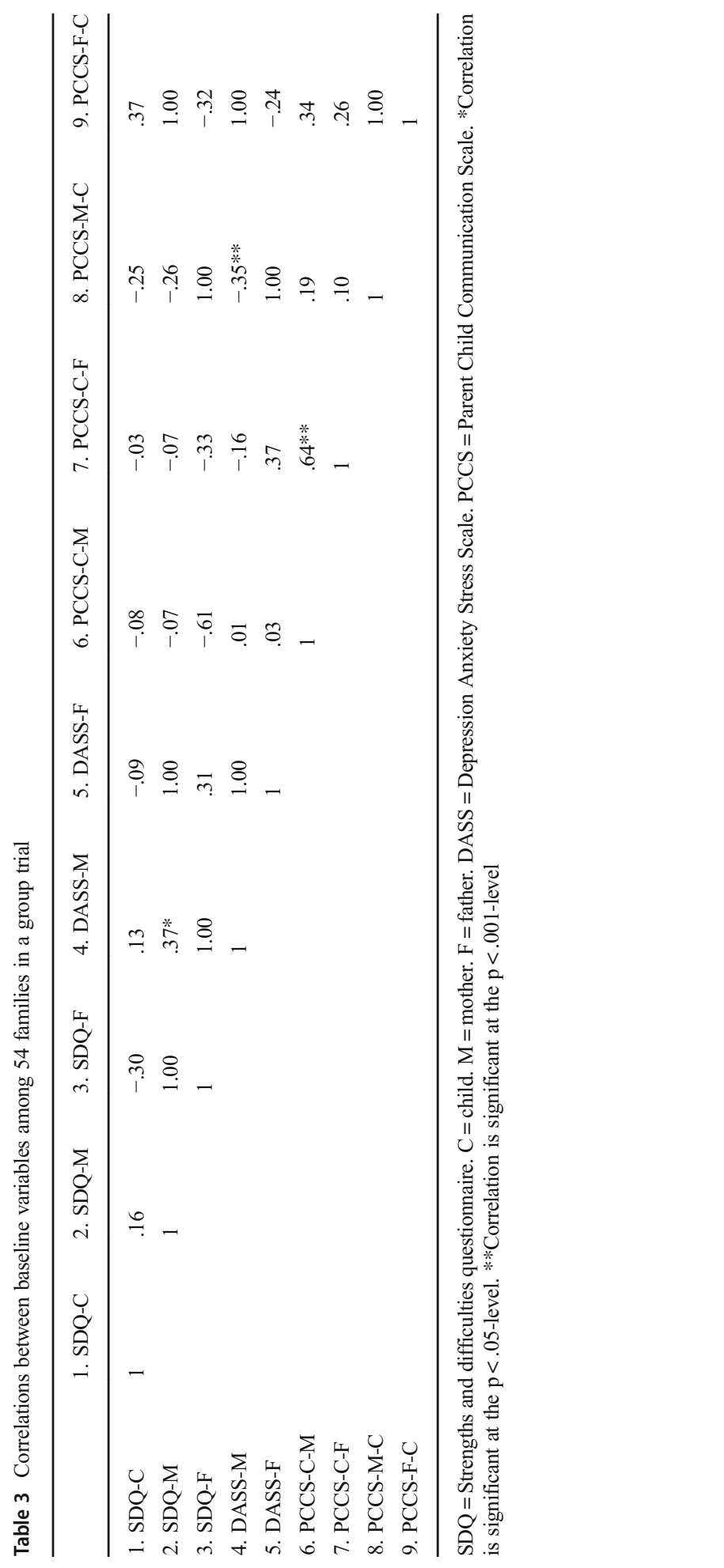


Table 4 Participant satisfaction with participating in groups

\begin{tabular}{|c|c|c|c|}
\hline Evaluation item & $\mathrm{M}(\mathrm{SD})$ & Possible range & Range \\
\hline \multicolumn{4}{|l|}{ Siblings } \\
\hline Overall evaluation & $3.4(0.8)$ & $1.0-4.0$ & $1.0-4.0$ \\
\hline How important was it to be in the group? & $13.1(2.3)$ & $0.0-16.0$ & $7.0-16.0$ \\
\hline How useful was it to be in the group? & $13.2(2.2)$ & $0.0-16.0$ & $7.0-16.0$ \\
\hline \multicolumn{4}{|l|}{ Parents } \\
\hline \multicolumn{4}{|l|}{ Perceived usefulness in terms of... } \\
\hline Increased competence in talking about the child's disorder & $3.8(0.5)$ & $0.0-4.0$ & $2.0-4.0$ \\
\hline $\begin{array}{l}\text { Increased insight for parents in sibling knowledge about } \\
\text { the disorder and its' challenges }\end{array}$ & $3.7(0.6)$ & $0.0-4.0$ & $2.0-4.0$ \\
\hline Learn a technique to work together on the child's challenges & $3.6(0.7)$ & $0.0-4.0$ & $2.0-4.0$ \\
\hline Increased parental competence in exploring siblings' challenges & $3.7(0.6)$ & $0.0-4.0$ & $2.0-4.0$ \\
\hline $\begin{array}{l}\text { Increased communication between parents and children } \\
\text { about sibling experiences }\end{array}$ & $3.7(0.7)$ & $0.0-4.0$ & $2.0-4.0$ \\
\hline
\end{tabular}

often); four (7.2\%) concerned more time to play (e.g., I want more time to play games); whereas the remaining were various comments (e.g., For me, this is important). There was only one comment that we understood as negative feedback (i.e., Please stop pressuring people to speak).

From 55 open-ended responses from caregivers about what should be done more or less (i.e., differently) in the groups, 17 (30.9\%) concerned participants' behavior and involvement in the group (e.g., We shouldn't hide the ideas we have in the mind), 16 (29.0\%) concerned group contents (e.g., Help to encourage parents to practice at home), ten (18.2\%) concerned wishing there was more time for the groups (e.g., Suggest to develop this program more often and wider); six (10.9\%) were general praise (e.g., I am so satisfied that teachers have developed this program (....) As mother, I am more aware about what I earlier didn't understand, and now I understood better than before), four (7.2\%) concerned new insights about the sibling (e.g., I must listen and teach him better); whereas the remaining were unclassifiable. None of the comments from caregivers were considered negative.

\section{Discussion}

This is the first study to examine the SIBS intervention in an Asian context. We first examined the levels of sibling and parent mental health, as well as family communication at baseline, in the current sample. Labelling the levels of these variables in terms of high versus low is tricky, due to lack of Cambodian (or indeed, South-East Asian) norm data. Notably, parents rated siblings' mental health scores as considerably higher than sibling self-report. This is in contrast to norm data from Britain and Australia, on which self-report is typically higher than parent report (Mellor, 2005; Meltzer et al. 2003). In the Norwegian SIBS trial, siblings also self-reported more mental health problems than parents (Haukeland et al. 2020). Although we do not know the reasons 
for the difference in sibling self-reported mental health and parent-reported mental health for siblings, discrepancy between child- and parent-report is common, and the norm rather than the exception in child mental health (De Los Reyes 2011). Nevertheless, it is important to consider why siblings in Cambodia rated their mental health significantly lower than how their parents rated the siblings' mental health. Although participation was voluntary for all, it is likely that parents were the main decision agents and chose to participate due to concerns about the sibling. The siblings may not have shared these concerns. Relatedly, parents are likely more used to Caritas-CCAMH due to bringing the child with ND to clinic visits, and may thereby have had a more "clinical mindset" when rating their children who are siblings. Another possible explanation is that siblings may have underreported their concerns, as research shows siblings of children with disorders want to protect their parents (Haukeland et al. 2015). The relatively high parent-rated mental health for siblings may also in part reflect parents' own mental health problems, although this influence appears limited due to the small correlations between parents rating of their own and their children's mental health. Further studies are needed to identify culturally sensitive and appropriate mental health measures for children in the Cambodian setting, and to establish norm data for Cambodia.

In terms of parental mental health, the mean scores of the current sample were high, and despite lack of available norm data, they nevertheless clearly indicate considerable mental health needs among the Cambodian caregivers. A recent review of stress among parents of children with autism spectrum disorders (ASD) in South-East Asia identified no studies from Cambodia (Ilias et al. 2018). However, across studies from other South-East Asian countries, the following predictors of parental mental health were identified: social support, financial difficulty, ASD severity, parental understanding of ASD, worries about the child's future, and religious beliefs (Ilias et al. 2018). It is reasonable to assume that at least some of these apply also in Cambodia. Our initial findings indicate considerable mental health needs among Cambodian caregivers of children with ND.

Another aspect to note about our findings is the lack of difference in parental mental health scores between mothers and fathers. This is in contrast to well-documented gender differences generally showing higher mental health problems for women than men (e.g., Ihlebæk et al. 2002; Mong and Cusmano 2016). It is also surprising given previous findings that mothers generally engage more in the upbringing and caretaking of the children with disabilities, worry more, and spend more time and energy than their spouses on taking care of the child (Dellve et al. 2006). Caution is required due to the low percentage of fathers in our sample, however our results indicate fathers' mental health is as poor as mother mental health - a particularly concerning finding that men are less likely than women to seek help and experience social support (Melchiorre et al. 2013).

In terms of family communication, scores were lower in the current Cambodian sample compared to a Norwegian sample using the same measure (Haukeland et al. 2020). These findings are hard to interpret, however, given the large cultural differences in family culture between these countries. Ethnographic research has shown that although the nuclear family remains the most common family structure in Cambodia, 
influenced both by Theravada Buddhism and influences through the French colonization, there is a tendency for more extended and flexible living arrangements due to economic changes and urbanization (Heuveline and Hong 2017). It is also still unclear how the extremity of the Pol Pot regime in the 1970s may have affected family beliefs in Cambodia. During the Khmer Rouge era, people were forced away from family members and a socio-cultural brainwash enforcing allegiance to the political structure of the regime, called Angkar, rather than to the family and elders was attempted (Heuveline and Hong 2016). In particular the younger generation were forced to move loyalty and bonds away from the family (Demont and Heuveline 2008). Such influences on the family may also have impacted on family communication natures, and communication models based on current Western cultures may not be applicable. More research, but also theory development, is needed to determine how family communication should best be operationalized in the Cambodian culture.

Our second aim was to examine initial outcomes of SIBS, when delivered in Cambodia. Despite large cultural differences between Cambodia and Norway, where the SIBS intervention was developed, results were promising for some outcomes. Parentrated mental health for siblings, as well as parent self-reported mental health significantly improved from baseline to 4-month follow-up. However, and importantly, the main component of the parent groups in SIBS, i.e., parent-child communication, remained unchanged based on sibling- and parent-report. Given that family communication significantly increased from baseline to 6-month follow-up in the Norwegian SIBS-trial (Haukeland et al. 2020), this indicates that the communication component of SIBS needs to be better tailored for the Cambodian culture. The practical experience from the group leaders running the intervention was also that sessions 3 and 5 were quite unsuccessful. Participants spent little time on this task and seemed to struggle with the task. It is worth reflecting on the reasons why this might have been the case. The purpose of the joint sessions may not have been sufficiently or clearly enough explained to participants.

The awkwardness of the task may also reflect structural differences between Cambodian and Norwegian family cultures. Whereas the Norwegian culture places a lot of value on the autonomy of adolescents, and encourages youth to challenge their parents (i.e., this is regarded a central developmental task), these values differ from Asian cultural ideals. In their theoretical framework, Ishikawa and Yamazaki (2005) elaborated on four cultural dimensions that are different in Western and Eastern cultures, with implications also for family communication patterns. The first dimension is individuality-collectivism. Whereas Western culture values individual rights and needs, Eastern cultures value the social context surrounding the individual more, emphasizing families as an entity more than the individual members. The second dimension is low context-high context. Whereas Western cultures are characterized by low-context communication, in which messages are typically direct and explicit, Eastern cultures tend to be more high-context, valuing indirect and non-verbal messages more than direct communication of feelings and opinions. The third dimension is masculinity-femininity. Western communication styles are regarded to value more typically masculine traits (e.g., assertive, competitive), whereas Eastern culture value typically feminine traits in communication more (e.g., cooperative, modest). The final dimension is Christianity-Confucianism. This dimension has to do with power structures. Whereas Western cultures are typically embedded in Christian (protestant-Lutheran) heritage 
emphasizing egalitarian relationships, Eastern cultures emphasize the Confucian ideals of harmony, obedience, and respect for seniors. Although descriptive, and not normative, these underlying cultural differences may help explain why the communication training principles developed in Norway do not seem to apply in the Cambodian context. A different starting point is needed to tailor the communication training in Cambodia.

In the current study, sibling self-reported mental health remained unchanged from baseline to follow-up. One potential reason is that sibling self-reported mental health was significantly lower than parent-reported mental health (for siblings) at baseline. Thus, there was less room for improvement from siblings' perspectives. Alternatively, and/or additionally, this indicates that the intervention needs to be further tailored for siblings in the Cambodian context to be effective.

Our final aim was to assess initial feasibility. User satisfaction was very high from the perspectives of siblings and parents. There was only one (slightly) negative comment in the open feedback form, and many siblings and parents noted more intervention time as an improvement point. Although this holds some promise in terms of user acceptability, there are also some concerns to note in terms of feasibility. We missed follow-up data from a quarter of the original participants. We cannot rule out that participants who did not show up for follow-up had poorer outcomes than those assessed. Note, however, that the dropout rate was almost half the sample, in the Norwegian trial (Haukeland et al. 2020). The high dropout rate in both countries may reflect the considerable strain facing the families of children with disabilities.

In future trials of the SIBS intervention, it is important to consider what can be done to reduce missing data at follow-up measurement points. In the ongoing randomized controlled trial of the SIBS intervention in Norway [Authors' own], all questionnaire data are collected electronically, meaning participants can log on via smart phones, tablets, and/or computers to respond at follow-up measurement points. This means no clinic attendance and no postage return is required. Reminders are sent at all measurement points via sms and/or email to participants. According to the most recent statistics of the Telecommunication Regulator of Cambodia (2020), Cambodia has 20.4 million mobile subscribers and 15.3 million internet subscribers. With an estimated 2020 population of 16.7 million people, the statistics indicate that most Cambodians have internet access. However, there is reason to believe the access is socio-economically skewed, and that online data collection may suit best as a supplement rather than the default setting currently.

The strengths of the current study include the novelty of the approach for a group with largely unmet service needs, and the inclusion of multi-informants and timepoints. However, there are several limitations. Due to practical and financial constraints, there was no official back-translation of the SIBS manual, meaning that the purpose of some of the contents may have been lost in translation. However, it is important to note that this study took place within an ongoing collaboration between a Norwegian team and the Caritas-CCAMH clinic, so there was considerable discussion about the contents and reflections made during team discussions. The relatively low reliability scores on some of the measures indicate that more research is needed to validate the questionnaires used for use in Cambodia.

We consider the current study an initial attempt to evaluate SIBS in Cambodia, and that further adaptations will be needed in the future. The lack of a control group means we cannot conclude that the observed changes are due to the SIBS intervention. We 
measured outcomes 4 months after the intervention, and only used evaluation forms (feasibility) immediately after. This was done to not inflate outcomes and to examine potential change after some time. However, the outcomes are trickier to interpret when conducted several months after intervention. We cannot rule out that other interventions have impacted outcomes, although to the best of our knowledge, there are no interventions available in Cambodia targeting siblings and/or family communication. We lost a quarter of the sample from baseline to follow-up, which further compromises generalizability of our results. Finally, the sample included a heterogeneous set of NDs, which limits our ability to specify the target groups for SIBS in much detail.

The main implication of the current study is that the SIBS manual should be further developed and evaluated in the Cambodian context. We will arrange a set of workshops with local stakeholders to discuss how to enhance the intervention. We will also conduct qualitative studies, and our long-term goal is to examine a Cambodian version of the manual in a randomized controlled trial. These are important goals, given our findings that both siblings and parents of children with ND in Cambodia experience mental health problems.

Funding Open Access funding provided by University of Oslo (incl Oslo University Hospital). The study was supported with travel grants from Uni Research, Bergen, Norway; The Regional Centre for Child and Adolescent Welfare Research, Bergen, Norway; and the Department of Psychology, University of Oslo, Norway. The National Advisory Unit for Rare Disorders in Norway funded the current project. Caritas supports the clinic in which the study was conducted.

\section{Compliance with Ethical Standards}

Conflicts of Interest/Competing Interests The authors declare that they have no conflicts of interest.

Ethical Approval The study was approved by the review boards for medical research ethics in Cambodia and Norway and with the 1964 Helsinki declaration and its later amendments or comparable ethical standards.

Informed Consent All caregivers provided informed consent to participate on behalf of themselves and their children.

Open Access This article is licensed under a Creative Commons Attribution 4.0 International License, which permits use, sharing, adaptation, distribution and reproduction in any medium or format, as long as you give appropriate credit to the original author(s) and the source, provide a link to the Creative Commons licence, and indicate if changes were made. The images or other third party material in this article are included in the article's Creative Commons licence, unless indicated otherwise in a credit line to the material. If material is not included in the article's Creative Commons licence and your intended use is not permitted by statutory regulation or exceeds the permitted use, you will need to obtain permission directly from the copyright holder. To view a copy of this licence, visit http://creativecommons.org/licenses/by/4.0/.

\section{References}

Clayton, T. (2017). Transition, culture, and language in Cambodia. In A. B. M. Tsui \& J. W. Tolefson (Eds.), Language policy, culture, and identity in Asian contexts. New York: Routledge (e-book). https://doi.org/ 10.4324/9781315092034-6.

Cohen, J. (1992). A power primer. Psychological Bulletin, 112(1), 155-159. https://doi.org/10.1037//00332909.112.1.155. 
De Los Reyes, A. (2011). More than measurement error: Discovering meaning behind informant discrepancies in clinical assessments of children and adolescents. Journal of Clinical Child and Adolescent Psychology, 40(1), 1-9. https://doi.org/10.1080/15374416.2011.533405.

Dellve, L., Samuelsson, L., Tallborn, A., Fasth, A., \& Hallberg, L. R. M. (2006). Stress and wellbeing among parents of children with rare diseases: A prospective intervention study. Journal of Advanced Nursing, 53(4), 392-402. https://doi.org/10.1111/j.1365-2648.2006.03736.x.

Demont, F., \& Heuveline, P. (2008). Diversity and change in Cambodian households, 1998-2006. Journal of Population Research, 25, 287-313. https://doi.org/10.1007/BF03033892.

Fisman, S., Wolf, L., Ellison, D., \& Freeman, T. (2000). A longitudinal study of siblings of children with chronic disabilities. Canadian Journal of Psychiatry, 45(4), 369-375. https://doi.org/10.1177/ 070674370004500406.

Giallo, R., Roberts, R., Emerson, E., Wood, C., \& Gavidia-Payne, S. (2014). The emotional and behavioural functioning of siblings of children with special health care needs across childhood. Research in Developmental Disabilities, 35(4), 814-825. https://doi.org/10.1016/j.ridd.2014.01.017.

Goodman, R. (2001). Psychometric properties of the strengths and difficulties questionnaire. Journal of the American Academy of Child and Adolescent Psychiatry, 40(11), 1337-1345. https://doi.org/10.1097/ 00004583-200111000-00015.

Grundy, J., Khut, Q. Y., Oum, S., Annear, P., \& Ky, V. (2009). Health system strengthening in Cambodia-A case study of health policy response to social transition. Health Policy, 92(2), 107-115. https://doi.org/10. 1016/j.healthpol.2009.05.001.

Hafting, M., Aadland, G., Pat, P., Fjermestad, K., \& Jegannathan, B. (2020). A Cambodian-Norwegian competence building program in child mental health: A qualitative evaluation. [Manuscript submitted for publication]. Center for International Health, University of Bergen.

Haukeland, Y. B., Fjermestad, K. W., Mossige, S., \& Vatne, T. M. (2015). Emotional experiences among siblings of children with rare disorders. Journal of Pediatric Psychology, 40(7), 712-720. https://doi.org/ 10.1093/jpepsy/jsv022.

Haukeland, Y. B., Fjermestad, K. W., Czajkowski, N. O., Mossige, S., Silverman, W. K., \& Vatne, T. M. (2020). Evaluation of a joint parent-child intervention for siblings of children with chronic disorders. Journal of Child and Family Studies, 29, 2201-2217. https://doi.org/10.1007/s10826-020-01737-x.

Heuveline, P., \& Hong, S. (2016). One-parent families in contemporary Cambodia. Marriage and Family Review., 52(1-2), 216-242. https://doi.org/10.1080/01494929.2015.1076553.

Heuveline, P., \& Hong, S. (2017). Household structure and child education in Cambodia. International Journal of Population Studies, 3(2), 1-15. https://doi.org/10.18063/ijps.v3i2.309.

Ihlebæk, C., Eriksen, H. R., \& Ursin, H. (2002). Prevalence of subjective health complaints (SHC) in Norway. Scandinavian Journal of Public Health, 30(1), 20-29. https://doi.org/10.1177/14034948020300010701.

Ilias, K., Cornish, K., Kummar, A, S., Park, M, S, A., \& Golden, K, J. (2018). Parenting stress and resilience in parents of children with autism Spectrum disorder (ASD) in Southeast Asia: A systematic review. Frontiers in Psychology, 9(280). https://doi.org/10.3389/fpsyg.2018.00280.

Ishikawa, H., \& Yamazaki, Y. (2005). How applicable are Western models of patient-physician relationship in Asia?: Changing patient-physician relationship in contemporary Japan. International Journal of Japanese Sociology, 14, 84-93. https://doi.org/10.1111/j.1475-6781.2005.00070.x.

Jegannathan, B., Kullgren, G., \& Deva, P. (2014). Mental health service in Cambodia: Challenges and opportunities in a post-conflict setting. Asian Journal of Psychiatry, 13, 75-80. https://doi.org/10.1016/ ajp.2014.12.006.

Karst, J. S., \& Van Hecke, A. V. (2012). Parent and family impact of autism spectrum disorders: A review and proposed model for intervention evaluation. Clinical Child and Family Psychology Review, 15(3), 247277. https://doi.org/10.1007/s10567-012-0119-6.

Knudsen, S. A. K., Overland, H. F., Aakvaag, S. B., Harvey, M., Hotopf, A., \& Mykletun. (2010). Common mental disorders and disability pension award: seven year follow-up of the HUSK study. European Journal of Public Health, 20, 25-25.

Lobato, D. J., \& Kao, B. T. (2002). Integrated sibling-parent group intervention to improve sibling knowledge and adjustment to chronic illness and disability. Journal of Pediatric Psychology, 27(8), 711-716. https:// doi.org/10.1093/jpepsy/27.8.711.

Loeber, R., Farrington, D. P., Stouthamer-Loeber, M., \& Van Kammen, W. B. (1998). Antisocial behavior and mental health problems: Explanatory factors in childhood and adolescence. Mahwah: Lawrence Erlbaum. 
Lovibond, P. F., \& Lovibond, S. H. (1995). The structure of negative emotional states: Comparison of the depression anxiety stress scales (DASS) with the Beck depression and anxiety inventories. Behaviour Research and Therapy, 33(3), 335-343. https://doi.org/10.1016/0005-7967(94)00075-u.

McKenzie Smith, M., Pinto Pereira, S., Chan, L., Rose, C., \& Shafran, R. (2018). Impact of well-being interventions for siblings of children and young people with a chronic physical or mental health condition: A systematic review and meta-analysis. Clinical Child and Family Psychology Review, 21(2), 246-265. https://doi.org/10.1007/s10567-018-0253-x.

Melander, M., Dahlblom, K., Jegannathan, B., \& Kullgren, G. (2016). Exploring communication of traumatic experiences from Khmer rouge genocide survivors to their offspring: In-depth interviews with both generations. International Journal of Social Psychiatry, 62(4), 327-333. https://doi.org/10.1177/ 0020764016631364.

Melchiorre, M. G., Chiatti, C., Lamura, G., Torres-Gonzales, F., Stankunas, M., Lindert, J., Ioannidi-Kapolou, E., Barros, H., Macassa, G., \& Soares, J. F. (2013). Social support, socio-economic status, health and abuse among older people in seven European countries. PLoS One, 8(1), e54856. https://doi.org/10.1371/ journal.pone.0054856.

Mellor, D. (2005). Normative data for the Strengths and Difficulties Questionnaire in Australia. Australian Psychologist 40(3), 215-222. https://doi.org/10.1080/00050060500243475.

Meltzer, H., Gatward, R., Goodman, R., \& Ford, T. (2003). Mental health of children and adolescents in Great Britain. International Review of Psychiatry, 15(1-2), 185-187. https://doi.org/10.1080/ 0954026021000046155.

Mong, J. A., \& Cusmano, D. M. (2016). Sex differences in sleep: Impact of biological sex and sex steroids. Philosophical Transactions of the Royal Society B: Biological Sciences, 371(1688), 20150110. https:// doi.org/10.1098/rstb.2015.0110.

Morgan, F., \& Tan, B.-K. (2011). Parental views from rural Cambodia on disability causation and change. Disability and Rehabilitation, 33(21-22), 2114-2120. https://doi.org/10.3109/09638288.2011.560334.

Murphy, L. K., Murray, C. B., \& Compas, B. E. (2017). Topical review: Integrating findings on direct observation of family communication in studies comparing pediatric chronic illness and typically developing samples. Journal of Pediatric Psychology, 42(1), 85-94. https://doi.org/10.1093/jpepsy/jsw051.

Phillips, R. S. C. (1999). Intervention with siblings of children with developmental disabilities from economically disadvantaged families. Families in Society, 80(6), 569-577. https://oi.org/10.1606/1044-3894.1781.

Rossiter, L., \& Sharpe, D. (2001). The siblings of individuals with mental retardation: A quantitative integration of the literature. Journal of Child and Family Studies, 10(1), 65-84. https://doi.org/10.1023/ A:1016629500708.

Smith, T., \& Perry, A. (2005). A sibling support Group for Brothers and Sisters of children with autism. Journal on Developmental Disabilities, 11, 77-88. http://oadd.org/wp-content/uploads/2016/12/ smithperry.pdf.

Sommerschild, H. (1998). Mestring som styrende begrep. In B. Gjærum, B. Grøholt, \& H. Sommerschild (Eds.), Mestring som mulighet i møte med barn, ungdom og foreldre (pp. 21-60). Oslo: Tano Aschehoug.

Telecommunication Regulator of Cambodia. (2020). Telecom Statistics. https://www.trc.gov.kh/.

Thapar, A., Cooper, M., \& Rutter, M. (2017). Neurodevelopmental disorders. Lancet Psychiatry, 4(4), 339346. https://doi.org/10.1016/s2215-0366(16)30376-5.

Tudor, M. E., \& Lerner, M. D. (2015). Intervention and support for siblings of youth with developmental disabilities: A systematic review. Clinical Child and Family Psychology Review, 18(1), 1-23. https://doi. org/10.1007/s10567-014-0175-1.

Vatne, T. M., Haukeland, Y. B., Mossige, S., \& Fjermestad, K. W. (2019). The development of a joint parentchild intervention for siblings of children with chronic disorders. Fokus på familien, 47(1), 20-35. https:// doi.org/10.18261/issn.0807-7487-2019-01-03 ER.

Vermaes, I. P. R., van Susante, A. M. J., \& van Bakel, H. J. A. (2012). Psychological functioning of siblings in families of children with chronic health conditions: A meta-analysis. Journal of Pediatric Psychology, 37(2), 166-184. https://doi.org/10.1093/jpepsy/jsr081.

Vostanis, P. (2006). Strengths and difficulties questionnaire: Research and clinical applications. Current Opinion in Psychiatry, 19(4), 367-372. https://doi.org/10.1097/01.yco.0000228755.72366.05.

WHO (2020a). Cambodia. https://www.who.int/countries/khm/en/

WHO (2020b). Norway. https://www.who.int/countries/nor/en/

Williams, P. D., Williams, A. R., Graff, J. C., Hanson, S., Stanton, A., Hafeman, C., Liebergen, A., Leuenberg, K., Setter, R. K., Ridder, L., Curry, H., Barnard, M., \& Sanders, S. (2003). A community- 
based intervention for siblings and parents of children with chronic illness or disability: The ISEE study. The Journal of Pediatrics, 143(3), 386-393. https://doi.org/10.1067/s0022-3476(03)00391-3.

World Bank (2020). The World Bank in Cambodia. https://www.worldbank.org/en/country/cambodia/overview

Publisher's Note Springer Nature remains neutral with regard to jurisdictional claims in published maps and institutional affiliations.

\section{Affiliations}

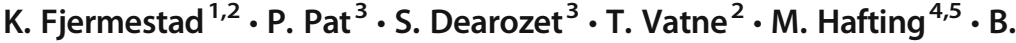 Jegannathan ${ }^{3}$}

1 Department of Psychology, University of Oslo, Oslo, Norway

2 Frambu resource centre for rare disorders, Siggerud, Norway

3 Caritas Child and Adolescent Mental Health Clinic, Chey Chumneas Referral Hospital, Thakmao, Cambodia

4 Voss Child and Adolescent Mental Health Services, Helse Bergen Regional Health Authority, Bergen, Norway

5 The Global Mental Health Research Group, Center for International Health, University of Bergen, Bergen, Norway 